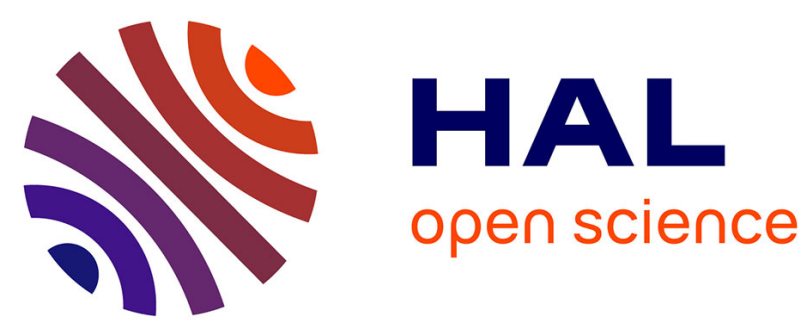

\title{
La problématique de l'insertion professionnelle des personnes présentant un handicap psychique: les différentes dimensions à prendre en compte
}

Bernard Pachoud, Alain Leplège, Arnaud Plagnol

\section{- To cite this version:}

Bernard Pachoud, Alain Leplège, Arnaud Plagnol. La problématique de l'insertion professionnelle des personnes présentant un handicap psychique: les différentes dimensions à prendre en compte. Revue française des affaires sociales, 2009, Handicap psychique et vie quotidienne, 1, pp.257-277. hal-01505706

\author{
HAL Id: hal-01505706 \\ https://hal.science/hal-01505706
}

Submitted on 11 Apr 2017

HAL is a multi-disciplinary open access archive for the deposit and dissemination of scientific research documents, whether they are published or not. The documents may come from teaching and research institutions in France or abroad, or from public or private research centers.
L'archive ouverte pluridisciplinaire HAL, est destinée au dépôt et à la diffusion de documents scientifiques de niveau recherche, publiés ou non, émanant des établissements d'enseignement et de recherche français ou étrangers, des laboratoires publics ou privés. 


\section{LA PROBLÉMATIQUE DE L'INSERTION PROFESSIONNELLE DES PERSONNES PRÉSENTANT UN HANDICAP PSYCHIQUE : LES DIFFÉRENTES DIMENSIONS À PRENDRE EN COMPTE} Bernard Pachoud, Alain Leplège, Arnaud Plagnol

La Documentation française | « Revue française des affaires sociales »

2009/1 | pages 257 à 277

ISSN 0035-2985

Article disponible en ligne à l'adresse :

http://www.cairn.info/revue-francaise-des-affaires-sociales-2009-1-page-257.htm

\section{Pour citer cet article :}

Bernard Pachoud et al., « La problématique de l'insertion professionnelle des personnes présentant un handicap psychique : les différentes dimensions à prendre en compte », Revue française des affaires sociales 2009/1 (), p. 257-277.

Distribution électronique Cairn.info pour La Documentation française.

(C) La Documentation française. Tous droits réservés pour tous pays.

La reproduction ou représentation de cet article, notamment par photocopie, n'est autorisée que dans les limites des conditions générales d'utilisation du site ou, le cas échéant, des conditions générales de la licence souscrite par votre établissement. Toute autre reproduction ou représentation, en tout ou partie, sous quelque forme et de quelque manière que ce soit, est interdite sauf accord préalable et écrit de l'éditeur, en dehors des cas prévus par la législation en vigueur en France. Il est précisé que son stockage dans une base de données est également interdit. 


\section{La problématique de l'insertion professionnelle des personnes présentant un handicap psychique : les différentes dimensions à prendre en compte}

Bernard Pachoud, Alain Leplège et Arnaud Plagnol*

\section{- Introduction}

L'insertion professionnelle des personnes présentant un handicap consécutif à des troubles psychiatriques, désormais qualifié de handicap psychique, est un sujet d'actualité pour un ensemble de raisons. L'évolution des soins psychiatriques en est une. Les progrès pharmacologiques liés notamment aux antipsychotiques de nouvelle génération, plus respectueux des performances cognitives que les traitements antérieurs, permettent mieux qu'auparavant d'envisager une reprise d'activité. Le raccourcissement des prises en charge hospitalières, rendu possible par ces nouveaux traitements, mais aussi par des contraintes d'économie de santé, conduit à ramener au plus tôt et à maintenir autant que possible les personnes dans leur environnement social, ce qui passe notamment par le maintien ou la restauration d'une activité, si possible à caractère professionnel. Un délai réduit entre l'interruption d'activité professionnelle du fait de la maladie et un éventuel retour à l'emploi est également un facteur facilitant la réinsertion. Sur le plan social, les lois sur le handicap et en particulier la récente loi de 2005, dont bénéficient maintenant fréquemment, au titre du handicap psychique, les personnes ayant présenté des troubles psychiatriques sévères, prévoient et favorisent des mesures de soutien à la réinsertion professionnelle. Corrélativement, se sont développées des structures d'aide à l'insertion professionnelle pour ces personnes, au sein desquelles se développe une expérience en matière d'évaluation et d'accompagnement vers l'emploi des «handicapés psychiques ».

Ces pratiques d'évaluation et de soutien à la réinsertion, dans lesquelles nous sommes nous-mêmes engagés en tant que psychiatres, ne seront ici que très brièvement présentées car tel n'est pas l'objectif de cet

\footnotetext{
* Bernard Pachoud : psychiatre, maître de conférences en psychopathologie à l'université de Paris-Diderot, chercheur au CREA (CNRS/École polytechnique).

Alain Leplège : psychiatre, professeur de philosophie à l'université Paris-Diderot.

Arnaud Plagnol : psychiatre, maître de conférences en psychopathologie à l'université Paris 8 , chercheur au LPN (Paris 8) et à l'IHPST (CNRS).
} 
article $^{1}$, et parce qu'elles restent hétérogènes. Ces pratiques se développent en France depuis une vingtaine d'années dans le secteur médicosocial, et leur diversité tient d'une part à l'histoire des établissements (certains étaient initialement orientés vers les soins, d'autres vers la formation, d'autres plutôt vers le travail dans un cadre "protégé»), d'autre part au parcours professionnel des intervenants de ces structures (cliniciens, formateurs, chargés d'insertion, plus rarement moniteurs d'ateliers professionnels). Schématiquement donc, ces pratiques se différencient par leur façon de pondérer l'importance accordée aux dimensions prises en compte, principalement la dimension clinique (repérage et prise en charge des freins ou obstacles personnels à la réinsertion), la dimension cognitive, et la dimension professionnelle (mise en situation de travail, attention portée aux contraintes du monde du travail, mais aussi aux ressources qu'il offre).

Ce qui nous frappe en tant que praticiens est que ce domaine d'activité, qui s'appuie essentiellement sur des pratiques, est encore peu théorisé. La même observation est d'ailleurs faite de façon récurrente (Liberman et al., 2005) à propos du contexte plus large de la réhabilitation psychosociale en psychiatrie. L'objectif de notre recherche résumée dans cet article, est donc de contribuer au développement d'un cadre théorique et de recherche qui permette l'analyse et l'évaluation de cette activité de soutien à la réinsertion, ainsi que son progrès.

Dans le contexte français, à défaut d'autres cadres théoriques disponibles pour cette activité d'évaluation et de soutien à la réinsertion professionnelle (et aussi parce qu'elle reste en grande partie assurée par des cliniciens), le cadre de référence dominant reste le cadre médical ou psychiatrique. Il semblait dès lors opportun de s'interroger sur l'existence d'autres cadres théoriques pertinents, permettant d'introduire d'autres points de vue, d'autres outils théoriques, et susceptibles ainsi d'enrichir ces pratiques.

Notre recherche s'est appuyée essentiellement sur une recension de la littérature académique traitant de l'insertion professionnelle des personnes ayant présenté des troubles psychiatriques graves, mais aussi plus largement sur des travaux consacrés à la notion de handicap psychique, à la réhabilitation psychosociale en psychiatrie, ainsi qu'aux enjeux psychologiques du travail.

Cet article ne prendra cependant pas tout à fait la forme classique d'une revue de la littérature pour une série de raisons. En premier lieu, notre domaine d'investigation - la problématique de la réinsertion professionnelle des personnes en situation de handicap psychique - est à la fois trop vaste pour permettre une revue complète de littérature, et cependant plus limité que des thématiques pourtant très pertinentes de la littérature, telles que celle

\footnotetext{
1. Nous tenons à remercier les «referees» dont les remarques critiques ont permis des clarifications dans notre texte, ainsi que Catherine des Moutis pour ses commentaires. Ce travail a été rendu possible par un séminaire de recherche pluridisciplinaire financé dans le cadre du programme de recherche de la Mire (Mission Recherche de la DREES) sur le handicap psychique (appel d'offre 2005), que les auteurs ont organisé en 2007 et 2008 sous le titre «Handicap psychique et réinsertion professionnelle».
} 
du handicap psychique, ou celle de la réhabilitation psychosociale ou des ressources permettant de surmonter le handicap.

En second lieu, notre objectif n'était pas de réunir les connaissances disponibles sur telle ou telle question bien définie relative à la réinsertion, mais d'identifier les cadres théoriques complémentaires au cadre médico-psychologique, et susceptibles de permettre d'enrichir nos pratiques de soutien à la réinsertion. Cela nous a conduits à rester à un certain niveau de généralité, et à faire parfois une présentation «orientée» de certains courants de recherche, dont nous n'avons retenu que les aspects nous semblant pertinents pour le handicap psychique et notre problématique de la réinsertion professionnelle. Nous n'avons développé que partiellement les modèles du handicap que nous invoquons, au risque de ne pas apporter tous les éléments nécessaires à leur complète intelligibilité. Enfin, les choix que nous avons faits, ont été orientés par notre enracinement dans une perspective médico-psychologique et notre souci de souligner l'intérêt et de promouvoir des approches différentes et complémentaires.

Notre intention initiale, confortée par l'examen de la bibliographie, était de faire droit au caractère pluridimensionnel du champ de recherche que constitue la problématique de l'insertion professionnelle des personnes présentant un handicap psychique. Cette pluridimensionnalité tient à la variété des facteurs qui jouent un rôle dans les possibilités d'insertion. Il importe, en effet, de tenir compte non seulement des facteurs directement liés à la personne (son état psychopathologique qui comporte lui-même des aspects pluridimensionnels, ses performances cognitives, ses compétences professionnelles, les facteurs motivationnels, ses aptitudes relationnelles ou sociales...), mais également des facteurs liés à la situation de travail (caractéristiques des tâches à accomplir, modes de coordination du collectif de travail, attendus de l'entreprise, modes de soutien envisageables...), le handicap étant toujours relatif à une situation, en l'occurrence aux exigences des tâches à accomplir et à l'environnement de travail.

Cette ambition d'envisager toutes les dimensions s'est cependant heurtée au fait que la littérature ne traite que très inégalement de ces dimensions. À cette difficulté s'ajoute celle d'une certaine hétérogénéité de cette littérature, liée à la variété des perspectives théoriques, des sensibilités disciplinaires, des modes d'approche de ces dimensions, ce qui témoigne certes de la richesse de la thématique, mais requiert en contrepartie de trouver un mode de structuration de ce champ de recherche qui, à défaut, peut paraître enchevêtré.

Nous nous limiterons ici à évoquer deux principales orientations de recherche. Elles n'épuisent pas la variété des travaux sur la réinsertion, mais nous les privilégierons car elles reflètent ce qu'on peut considérer comme le mouvement principal caractérisant l'évolution de la recherche sur le handicap psychique et la réinsertion depuis quelques décennies : cette évolution se caractérise par le développement, à côté du modèle médical de la maladie et de ses conséquences, d'un modèle du handicap alternatif au modèle médical, et à ce titre lui étant véritablement complémentaire. 
Nous présenterons donc d'abord les recherches empiriques portant sur les facteurs prédictifs de la réinsertion au travail, ou plus généralement sur l'évaluation du retentissement fonctionnel des pathologies psychiatriques. Ces recherches conduisent à reconnaître que l'évaluation psychopathologique et cognitive est insuffisante à l'appréciation des possibilités de réinsertion, ce qui ouvre la question des autres dimensions à prendre en compte, et des compétences requises pour les appréhender. Il est donc frappant de constater que ces recherches, bien que menées essentiellement dans un cadre médical, aboutissent à souligner la nécessité de recourir à des approches complémentaires, pour appréhender des dimensions inaperçues ou négligées par l'approche médicale. Peut-être, peut-on y voir l'influence indirecte des modèles non médicaux du handicap.

Nous aborderons ensuite les modèles non médicaux du handicap psychique, à partir de contributions retenues dans l'importante littérature sur la notion de handicap psychique ainsi que sur les facteurs et ressources aidant à surmonter ce handicap (ces deux thématiques, handicap psychique et moyens de le surmonter, sont le plus souvent associées dans ces travaux). Rappelons d'abord que l'intérêt des théorisations du handicap, relativement à l'approche classiquement médicale des troubles, est lié au déplacement d'attention de la maladie vers ses conséquences pour la personne, puis dans un second temps au déplacement d'attention des déterminants individuels ou personnels de ces conséquences vers leurs déterminants situationnels ou environnementaux. Pour rendre pleinement intelligible l'originalité de ces approches non médicales du handicap, il convient de comprendre les conditions de leur émergence en les rapportant à leur histoire sociale et politique (l'histoire des mouvements de personnes handicapées). Les modèles dits sociaux du handicap ont été développés par les mouvements de personnes handicapées, dans les pays anglo-saxons, pour s'opposer au «modèle médical » et aux pratiques de prise en charge qui leur étaient imposées (réadaptation et rééducation médicale, institutionnalisation...). C'est ainsi que doit être comprise l'opposition d'un modèle médical et individuel du handicap à un modèle social du handicap : social car le handicap y est conçu comme la conséquence des obstacles posés par la société aux personnes présentant une déficience (ce sont dès lors ces circonstances matérielles ou sociales qui doivent être amendées), alors que dans le modèle médical, le handicap est conçu comme conséquence d'un déficit ou d'une incapacité de l'individu, qui doit par conséquent s'adapter.

Nous ne développerons pas d'avantage ici ce déplacement de perspective sur le handicap introduit par ces courants militants, et n'aborderons pas non plus les difficultés posées par l'application de ces approches nouvelles du handicap au cas particulier du handicap psychique. Ce contexte d'évolution des idées sur le handicap aide cependant à mieux comprendre les approches actuelles du handicap psychique, qui en général portent aussi sur les facteurs et ressources permettant de surmonter ce handicap, et dans lesquelles il est facile de reconnaître l'influence des approches non médicales 
du handicap. Le courant anglo-saxon de la réhabilitation psychosociale peut être considéré comme l'une des principales formes qu'a prise, pour le handicap psychique, l'approche nouvelle du handicap : l'accent est mis non sur les troubles résiduels de la personne ou ses déficiences, mais sur ses ressources pour surmonter les conséquences de la maladie. Les facteurs environnementaux sont également considérés, en particulier la stigmatisation qui frappe les troubles psychiatriques, de même que l'importance des étayages familiaux et sociaux. Cependant l'accent est mis moins sur ces déterminants situationnels, que sur les ressources de l'individu pour surmonter ses difficultés (quelle qu'en soit l'origine, de ses déficiences ou de la société). À ce titre, cette approche, si elle est bien complémentaire à un modèle médical des troubles, reste une approche privilégiant les déterminants individuels de la réinsertion plutôt que les déterminants environnementaux : autrement dit, elle relève encore d'un modèle individualiste du handicap plus que d'un modèle social tel que nous l'avons défini.

Nous n'avons pas repéré de courant important dans la littérature scientifique contemporaine qui privilégie véritablement un modèle social du handicap psychique. Il est possible que cette situation évolue. Remarquons cependant que la spécification et la compensation des déterminants situationnels du handicap psychique sont moins faciles à concevoir que dans le cas de certains handicaps sensoriels ou moteurs (avec par exemple la facilitation des accès pour les handicapés en fauteuil ou la signalisation tactile ou auditive pour les malvoyants). C'est certainement un des axes de recherche qui reste à développer pour le handicap psychique.

Le plan de notre article sera donc le suivant :

- Présentation des recherches empiriques sur les déterminants du retentissement fonctionnel des troubles psychiatriques.

- Intérêt d'un modèle propre du handicap psychique et des ressources pour le surmonter, distinct et complémentaire du modèle médical.

\section{- Recherches empiriques sur les déterminants du retentissement fonctionnel des troubles psychiatriques}

La richesse de la bibliographie sur la réinsertion professionnelle ${ }^{1}$ des malades mentaux manifeste l'actualité de cette thématique, en particulier dans le champ des recherches empiriques développées majoritairement dans le monde anglo-saxon. L'ampleur qu'a déjà prise ce courant dans les revues scientifiques internationales de psychiatrie témoigne d'un élargissement des

1. Voir également dans le présent numéro, l'article : «Déficits cognitifs et difficultés d'insertion professionnelle chez des personnes présentant une schizophrénie» de Marie-Noëlle Levaux, Isabelle Offerlin-Meyer, Frank Larøi, Martial Van der Linden et Jean-Marie Danion (ndlr). 
préoccupations scientifiques des psychiatres, et sans doute aussi d'une véritable transformation de la conception des maladies mentales. Elles semblent désormais considérées comme des maladies chroniques évolutives, dont le retentissement fonctionnel constitue une dimension essentielle; l'optimisation des conditions et de la qualité de vie de ces personnes devenant l'objectif premier de la politique de prise en charge. Une autre raison de s'intéresser à ces études est que leurs résultats sont parfois inattendus, du moins au regard de présupposés inhérents aux conceptions psychiatriques dominantes, et qu'elles ont par ailleurs des conséquences concrètes pour les pratiques d'évaluation.

Nous nous limiterons aux études portant spécifiquement sur les facteurs conditionnant la réinsertion professionnelle des schizophrènes, mais elles s'intègrent dans un domaine de recherche plus large et lui aussi nouveau, visant à évaluer de façon qualitative et surtout quantitative les conséquences fonctionnelles (functional outcomes) des pathologies psychiatriques (nous utiliserons ensuite plutôt le terme de «retentissement fonctionnel», bien qu'il ait une connotation certainement plus médicale que celui d'«outcome»).

Une première question est de savoir en quoi consiste au juste ce «retentissement fonctionnel », dans quels aspects de la vie quotidienne et des activités concrètes des personnes il se manifeste. Sans entrer dans le détail de cette question importante, et qui nous a paru étonnamment peu traitée dans la littérature, nous nous contenterons d'indiquer les quatre principales dimensions mentionnées dans les travaux de recherche pour apprécier ce retentissement fonctionnel. Il s'agit de :

- la qualité de vie subjective;

- l'insertion professionnelle;

- l'étendue et la qualité des relations sociales (ou de la vie relationnelle);

- l'autonomie ou l'indépendance dans la vie quotidienne.

Ces dimensions ne sont d'ailleurs pas définies autrement qu'opérationnellement, par des échelles d'évaluation qui leur sont consacrées ou, pour l'insertion professionnelle, par des données statistiques sur le taux d'emploi et sa durée moyenne pour la population considérée.

Ces travaux visent donc à spécifier ce retentissement fonctionnel, en en appréciant quantitativement l'importance ou la sévérité. Ils cherchent ensuite à l'identifier et à hiérarchiser ses principaux déterminants. L'objectif qui motive ces recherches est de pouvoir évaluer les pratiques, elles aussi en plein développement, qui visent à compenser, à pallier ce retentissement fonctionnel ou à y remédier (méthodes psycho-éducatives ou de réhabilitation psychosociale). Il s'agit enfin de mieux fonder ces pratiques, d'optimiser leur efficacité en leur permettant de cibler les principaux déterminants de ces effets invalidants. Or, pour apprécier l'efficacité d'une pratique, il convient de privilégier une évaluation quantitative, permettant une «mesure» de l'intensité de ce retentissement fonctionnel et de son évolution, après telle ou telle intervention de soutien. 


\section{Trois dimensions de la maladie mentale susceptibles de quantification : la symptomatologie, le déficit cognitif et le retentissement fonctionnel}

Il faut rappeler tout d'abord que ce type d'approche «quantitative», qui domine incontestablement la recherche objectivante en psychopathologie, et plus largement toute la psychiatrie académique contemporaine, a été initialement développé pour quantifier l'intensité des symptômes et apprécier l'efficacité sur ces derniers des traitements pharmacologiques. Il est maintenant très largement admis qu'une des meilleures façons de caractériser des troubles psychiatriques est de procéder à une évaluation qualitative et quantitative des manifestations symptomatiques de cet état.

Cette approche quantitative s'est également développée, ces dernières années, avec la prise en compte des troubles cognitifs qui accompagnent très souvent les pathologies psychiatriques. Or l'évaluation des troubles cognitifs se prête particulièrement à une approche quantitative, puisqu'il s'agit de mesurer l'altération des performances pour un certain nombre de fonctions cognitives (mnésique, attentionnelle, exécutive, communication verbale...).

On peut donc, aujourd'hui, caractériser assez précisément un état psychiatrique par une évaluation quantitative de ces deux dimensions, symptomatologique et cognitive. Les recherches sur le retentissement fonctionnel de la maladie, développées également en privilégiant une évaluation quantitative, contribuent de ce fait à définir une troisième dimension, qui apparaît naturellement comme complémentaire des précédentes.

La question qui, dès lors, ne peut manquer de se poser est celle du type de rapport entre ces trois dimensions. Sont-elles étroitement corrélées, de sorte que des troubles importants au niveau des symptômes s'accompagneraient d'altérations d'importance équivalente au niveau cognitif et, par suite, d'un retentissement fonctionnel proportionnel ? Y a-t-il des rapports de dépendance causale entre ces dimensions, au sens où, par exemple, la dimension des symptômes et celle des troubles cognitifs détermineraient le retentissement fonctionnel?

\section{La surprenante faiblesse de corrélation entre les symptômes, les troubles cognitifs et le retentissement fonctionnel, indice d'une relative indépendance de ces trois dimensions}

Contrairement à ce qu'on pourrait attendre, les recherches que nous présentons infirment dans une large mesure, l'idée d'une telle corrélation entre ces trois dimensions, et remettent par conséquent en cause la conception implicite de la pathologie psychiatrique qui conduit à l'imaginer. C'est un des intérêts de ces résultats et, pour le souligner, nous commencerons par 
préciser cette conception implicite des troubles psychiatriques et de leurs effets, conception à notre avis répandue parce qu'elle est justement dérivée des modes d'objectivation quantitatifs actuellement privilégiés.

L'évaluation quantitative des symptômes apparaît naturellement comme un bon indice de la sévérité de la pathologie, ce qui suggère une étroite corrélation entre les symptômes et le retentissement fonctionnel. Des scores élevés au plan des symptômes témoignent d'une forme grave de la maladie et à ce titre laissent envisager un retentissement fonctionnel important. En ce qui concerne les troubles cognitifs, dont l'importance est également quantifiée, dans la mesure où ils altèrent des fonctions «instrumentales » (fonction de communication, fonction mnésique, fonction exécutive...), il semble aller de soi que ces déficits ne peuvent qu'avoir un retentissement, d'un point de vue fonctionnel, sur la vie quotidienne et les activités concrètes des sujets. Par ailleurs, ces perturbations cognitives ont souvent été considérées comme susceptibles d'expliquer certains symptômes. On aboutit ainsi à une conception schématique selon laquelle le retentissement fonctionnel de la maladie serait déterminé essentiellement :

- par les symptômes (dont l'évaluation quantitative serait le reflet de la gravité de la pathologie);

- par des troubles cognitifs (dont la quantification serait un autre indicateur de la sévérité de la maladie).

Or cette conception se révèle être un préjugé, largement invalidé par les études actuelles de corrélation. Dans le cas de la schizophrénie, il apparaît en effet que, contrairement au présupposé courant, les troubles cognitifs n'expliquent pas les symptômes - pas plus d'ailleurs les symptômes positifs que les symptômes négatifs - (cf. : Zakzanis et Heinrichs, 1999; Heaton et al., 2001; Hughes et al., 2002; Addington et al., 1991; Bilder et al. 1985). Il apparaît d'autre part que les symptômes positifs (phénomènes hallucinatoires, idées délirantes, désorganisation de la pensée et du discours), bien qu'ils soient un bon indice de la sévérité des troubles, n'expliquent pas le retentissement fonctionnel (Strauss, 1993). La symptomatologie négative en revanche, bien que moins spécifique, du fait de ses effets globalement invalidants (ralentissement, altération de la motivation, voire de la volonté, appauvrissement de l'activité) est, en partie au moins, corrélée au retentissement fonctionnel ( $c f$. Addington et al., 1991; Cuesta et al., 1995 ; Morris et al., 1995 ; Summerfelt et al., 1991; Tamlyn et al., 1992). Quant aux troubles cognitifs nombreux et importants chez les schizophrènes, seuls certains d'entre eux seraient corrélés au retentissement fonctionnel (en l'occurrence, les troubles des fonctions exécutives et les troubles de la mémoire verbale) ( $c f$. Green, 1996; Meltzer et Mc Cgurk, 1999; Lysaker et al., 1995).

En résumé, dans le cas de la schizophrénie, et si on se limite à la question de l'insertion professionnelle (parmi les indicateurs du retentissement fonctionnel), il se dégage de ces études que, seuls la symptomatologie négative et certains des troubles cognitifs (la mémoire verbale et les fonctions exécutives) constituent des facteurs prédictifs de l'aptitude au travail. En revanche, 
d'autres facteurs qui ne sont ni des symptômes ni des déficits cognitifs, se révèlent des déterminants importants des possibilités de réinsertion professionnelle. Parmi ces facteurs, certains auteurs invoquent le niveau de formation scolaire et professionnelle antérieur à la maladie, ainsi que de l'expérience acquise en milieu professionnel avant la maladie (McGurk et Meltzer, 2000).

La thèse générale qui nous semble finalement pouvoir être dérivée de ces études est que, si seuls certains troubles cognitifs et une partie seulement de la symptomatologie participent au retentissement fonctionnel, en restant loin de suffire à l'expliquer, il est préférable d'admettre une relative indépendance de ces trois grandes dimensions de la pathologie psychiatrique (celle des symptômes, celle des troubles cognitifs, enfin, celle du retentissement fonctionnel). Une conséquence concrète pour les pratiques d'évaluation est que l'appréciation du retentissement fonctionnel (du handicap social, ou du handicap au travail) ne peut être inférée ou dérivée de l'évaluation clinique et cognitive. Il convient d'apprécier indépendamment, et comme une dimension propre, le retentissement fonctionnel. Cela suppose d'identifier les autres paramètres et dimensions qui jouent un rôle dans la réinsertion. Il n'est dès lors plus certain que les cliniciens soient les plus qualifiés pour effectuer cette appréciation.

Au-delà de leurs résultats, ces études illustrent un point sur lequel nous souhaitons attirer l'attention. Si la question de la réinsertion est intrinsèquement pluridimensionnelle, l'abord de cette question sera nécessairement dépendant du point de vue du chercheur (ou du praticien), de sa compétence disciplinaire, ce qui constitue un inévitable biais dans la recherche. Pour les cliniciens à l'origine des études que nous venons de commenter, il semble tenu pour acquis et inquestionné que les paramètres psychopathologiques et les performances cognitives doivent être les premiers facteurs à considérer avec cette population, quitte à admettre pour finir que d'autres dimensions méritent sans doute d'être aussi considérées. Si ces recherches sur les facteurs prédictifs du retour à l'emploi étaient entreprises par des psychologues du travail ou des sociologues, il est peu probable qu'ils partiraient des mêmes présupposés et commenceraient par apprécier l'incidence des facteurs psychopathologiques et cognitifs.

Ce biais de perspective dans l'abord de la réinsertion professionnelle est sans doute inévitable, mais impose alors de promouvoir une réelle approche pluridisciplinaire, non seulement pour la recherche, mais aussi pour la pratique du soutien à la réinsertion.

\section{Les facteurs prédictifs du retour à l'emploi : le rôle prépondérant des paramètres motivationnels et liés au travail, ainsi que des aptitudes relationnelles}

De façon pertinente pour nos pratiques d'évaluation du potentiel de réinsertion, il se dégage donc de ces études, que les facteurs prédictifs du retour à 
l'emploi ne sont pas prioritairement l'état psychopathologique, ni même le niveau de performance cognitive. Ce constat est d'ailleurs conforme à l'expérience clinique concernant l'adaptation au travail en milieu protégé des personnes présentant un handicap psychique ( $c f$. infra).

D'autres facteurs et dimensions doivent donc être considérés, qu'il importe désormais de spécifier. À partir de la bibliographie, mais aussi de notre propre expérience en matière de réinsertion professionnelle, nous pouvons évoquer au moins les facteurs suivants :

- des facteurs liés au rapport qu'entretient le sujet au travail, qui dépendent de l'expérience professionnelle acquise (et donc de l'histoire du sujet), des succès ou échecs rencontrés au travail, des attentes et de la valeur accordée au travail, enfin de la durée de l'interruption d'activité. (Corbière et al., 2002, 2004; Charbonneau, 2002);

- des facteurs motivationnels incluant le «sentiment d'efficacité personnelle à trouver un emploi, à s'y adapter, à surmonter ses difficultés psychiques (Bandura, 1997; Charbonneau, 2002);

- des enjeux de reconnaissance sociale (ou d'identité), qui interfèrent de façon souvent majeure dans le parcours d'insertion, mais de façons diverses, tantôt comme un facteur de motivation, tantôt comme un facteur d'inhibition (par peur de l'échec), ou enfin par leur incidence sur les choix d'orientation; - des capacités relationnelles, de l'ordre de la compétence sociale, qui s'avèrent un paramètre clé de l'employabilité;

- enfin il est tout à fait possible que d'autres facteurs, négligés ou mal appréhendés, jouent également un rôle dans le retour à l'emploi et restent à établir.

Concrètement, cela signifie que l'évaluation des possibilités de retour à l'emploi ne peut se limiter à, ni même être prioritairement, une évaluation psychopathologique. Contrairement à la croyance répandue que la sévérité du handicap est corrélée de façon linéaire à la sévérité de la maladie (voire à la sévérité seulement réputée de la maladie), l'expérience clinique comme les recherches empiriques montrent que le diagnostic n'est pas un facteur déterminant de la réinsertion (Charbonneau, 2002). Il reste toujours un peu frappant de constater, dans notre pratique, que les personnes psychotiques, notamment les schizophrènes, pathologie réputée pourtant fortement invalidante, ne sont pas les plus difficiles à réinsérer professionnellement. (Il est vrai que les progrès récents des traitements pharmacologiques ont modifié le pronostic fonctionnel de cette maladie.) Les personnes ayant des pathologies réputées moins lourdes, mais qui retentissent sur leurs capacités relationnelles, sur leur faculté de prise en compte de l'autre et d'adaptation à la relation, du fait de troubles anxieux ou névrotiques graves, notamment à forme obsessionnelle et compulsive, peuvent s'avérer très inadaptées au travail, en premier lieu pour ces raisons relationnelles qui les rendent inintégrables au collectif de travail, à quoi s'ajoutent des troubles de l'attention et de la concentration que génère l'angoisse. L'inadaptation au travail de ces personnes n'implique d'ailleurs pas nécessairement leur inadaptation sociale (elles sont souvent moins isolées que les schizophrènes par exemple, 
parvenant mieux à conserver un réseau relationnel, familial et social), ce qui illustre le fait que la notion de handicap est en réalité relative à un environnement ou à un contexte d'action. (Ces personnes peuvent être handicapées au travail sans l'être pour autant dans d'autres aspects de leur vie sociale; ce qui peut être l'inverse pour un schizophrène.)

En somme, on a sans doute encore insuffisamment pris la mesure de la profonde différence entre l'évaluation d'un état psychopathologique, l'évaluation du handicap (relativement au travail) lié à cette pathologie, et enfin l'évaluation des possibilités de surmonter ce handicap d'origine psychique. Cette difficulté tient pour une part au moins à la méconnaissance de la dimension propre du handicap, qui reste encore trop compris sur le modèle médical. L'enjeu est donc de développer un modèle propre du handicap psychique et des façons de le surmonter, qui ne soit pas calqué sur le modèle médical (psychiatrique) de la maladie à l'origine du handicap.

On peut ajouter que cette relative indépendance du retentissement fonctionnel, relativement aux symptômes et aux facultés cognitives, n'est surprenante que parce qu'elle invalide les présupposés liés à une certaine conception contemporaine de la psychopathologie. Elle n'est en revanche guère surprenante pour les cliniciens engagés dans des pratiques de réinsertion au travail des psychotiques. Il est d'expérience courante, en établissement de service et d'aide par le travail (ESAT) par exemple, que l'insertion au travail est souvent moins problématique pour des psychotiques conservant une symptomatologie résiduelle (hallucinations et idées délirantes), que pour d'autres mieux stabilisés au plan des symptômes mais ambivalents à l'égard des contraintes du travail. Autrement dit, ces études empiriques ne font que confirmer, en l'objectivant, ce qui est en réalité connu des cliniciens compétents, l'avantage de ces études étant de rendre généralisable, et plus facilement diffusable, ce savoir dont il s'agit alors de tirer toutes les conséquences.

Au plan de la prise en charge du retentissement fonctionnel de la maladie, que se propose d'assurer le courant de la réhabilitation psychosociale, il devient clair que les approches qui seraient seulement soucieuses de la réduction des symptômes (par le traitement psychotrope) et d'une compensation des déficits cognitifs resteront insuffisantes, faute de prendre en compte des paramètres qui pourraient bien, dans certains cas au moins, s'avérer les plus décisifs au plan fonctionnel. Cette remarque vaut en particulier au moment où le développement des techniques de réhabilitation psychosociale s'appuie, semble-t-il, prioritairement sur des outils de remédiation cognitive. Il est d'ailleurs tout à fait possible que l'efficacité des méthodes de réhabilitation cognitive, ne tienne pas seulement à la restauration ou au renforcement d'une fonction instrumentale, mais à des effets plus globaux tels que le renforcement de la confiance en soi, d'un sentiment d'efficacité.

Il ne s'agit pas de contester l'intérêt de ces approches, mais de les situer dans une perspective globale dans laquelle elles s'intègrent mais ne sauraient suffire. 
Il est notable que l'exigence d'une telle appréhension globale de la personne (ou de la situation de la personne handicapée) soit justement revendiquée par les travaux portant spécifiquement sur l'évaluation du handicap. Nous pensons en particulier à l'étude du Cédias pour la CNSA, Handicap d'origine psychique et évaluation des situations (Barreyre et al., 2007, 2009) ${ }^{1}$. L'intérêt de ces travaux est de spécifier précisément les facteurs et dimensions à prendre en compte dans une évaluation du handicap, et d'en proposer une liste qui est assez remarquablement complète. Barreyre et Makdessi (2007) spécifient les «éléments de connaissance» à réunir pour évaluer une situation de handicap d'origine psychique. Ils distinguent :

- les éléments dont on devrait disposer avant l'évaluation : le diagnostic; le projet de vie (ou les choix de vie au sens d'Amartya Sen de capabilités); le fonctionnement cognitif; le parcours de vie (scolarité, vie professionnelle, vie familiale, événements marquants);

- et ceux qu'il s'agit de réunir lors de l'évaluation : le rapport à la maladie ; les compétences sociales; et enfin les ressources personnelles et/ou propres à l'entourage (estime de soi, position sociale et rôles, réseaux de soutien social, familial ou professionnel...).

On retrouve la même exigence de pluridimensionnalité dans les travaux du réseau Galaxie ${ }^{2}$, en particulier dans son élaboration d'une "grille d'évaluation » pour les «Équipes spécialisées dans l'evaluation du handicap psychique » (Vidal-Naquet et al., 2007). Sont retenus et distingués cinq grands domaines devant faire l'objet d'une évaluation : le champ des processus cognitifs; le champ des habiletés psychomotrices et professionnelles; le champ des habilités sociales, le champ de l'identité personnelle (qui comporte la perception de soi et de ses compétences, la gestion émotionnelle, les habiletés décisionnelles, la sensibilité au changement, et l'adaptation au collectif); et enfin le champ de la psychopathologie.

On constate donc que ces deux guides d'évaluation, loin de s'en tenir à l'évaluation psychopathologique ou des performances cognitives, s'appliquent à spécifier les autres dimensions de l'évaluation, en particulier le projet de vie ou des choix de vie; le parcours de vie; les habilités sociales; le rapport à la maladie; et enfin les ressources personnelles ou propres à l'entourage.

Il est donc notable que ces études échappent au biais que nous dénonçons chez les cliniciens, consistant à privilégier les dimensions psychopathologiques et cognitives. C'est bien ce qui fait, à notre sens, la qualité de leurs travaux, mais dans les deux cas, cette conception «globale» de l'évaluation du handicap, reposant sur une évaluation expressément pluridimensionnelle, a été proposée par des chercheurs issus des sciences sociales et non de la médecine.

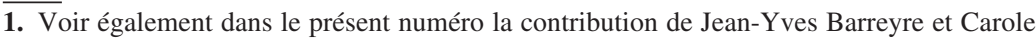
Peintre : «Les conditions de l'évaluation des situations de handicap d'origine psychique» (ndlr). 2. Voir également dans le présent numéro la contribution de Roger Gayton et Yann Boulon : «L'évaluation du handicap d'origine psychique et des besoins de compensation» (ndlr). 
Dès lors la question qui demeure est celle des compétences disciplinaires requises pour réaliser ces évaluations réellement pluridimensionnelles. Dans les deux cas, les auteurs insistent sur la nécessaire pluralité des partenaires de cette évaluation, dont le caractère global résulte d'une complémentarité disciplinaire. Une autre interprétation de l'évaluation globale pourrait être de l'assimiler à l'adoption d'une posture clinique, si on entend par là cette ouverture à la pluridimensionnalité indéfinie de la situation de chaque personne. Une telle interprétation, qui confie au seul «clinicien» la responsabilité d'une appréhension globale, s'expose à nouveau au risque d'une évaluation déséquilibrée dans la prise en compte des dimensions pertinentes, privilégiant la dimension psychopathologique.

\section{- Intérêt d'un modèle propre du handicap psychique, distinct et complémentaire du modèle médical}

Certaines approches de la réinsertion sociale des malades mentaux ont en commun de contribuer, explicitement ou implicitement, au développement d'une conception ou d'un modèle autonome du handicap, affranchi du modèle médical : modèle de type social, au sens que nous avons évoqué dans l'introduction (c'est-à-dire insistant sur les déterminants situationnels du handicap) ou modèle centré sur les enjeux existentiels du «vivre avec un handicap » et sur les ressources pour dépasser le handicap. Le courant anglosaxon de la réhabilitation psychosociale, que nous allons présenter, correspond à ce dernier type de modèle (centré sur les ressources pour surmonter le handicap), mais on retrouve des thèses convergentes dans la conception du handicap associée aux travaux sur la résilience, ou dans les travaux de l'OMS préparatoires à la Classification internationale du fonctionnement, du handicap et de la santé (que, faute de place, nous ne développerons pas ici). L'intérêt d'un tel modèle spécifique du handicap, distinct du modèle médical, est de permettre la prise en compte, voire la découverte, de dimensions importantes pour «surmonter» le handicap, qui restent négligées ou méconnues par la perspective médicale (centrée sur l'évaluation psychopathologique ou cognitive); dimensions qu'il importe dès lors d'intégrer parmi les facteurs de la réinsertion.

L'importance accordée dans ces approches à la dimension expérientielle - c'est-à-dire au vécu subjectif du handicap, mais aussi aux choix qu'il revient à la personne de faire en fonction de ses valeurs, à ses décisions en fonction de ses projets de vie - conduit à admettre que la connaissance de ces facteurs ne relève pas d'une méthodologie objectivante, en troisième personne, mais requiert une prise en compte de ce vécu et par conséquent une co-construction de ce savoir avec les usagers, dont le point de vue «en première personne» est requis. Il est possible que cette contrainte épistémologique soit parmi les raisons du retard dans le développement et la 
reconnaissance académique de ces approches, en France en particulier, voire de la résistance (passive) qu'elles suscitent.

\section{Une propriété du handicap psychique : la réversibilité des rapports de dépendance entre la maladie, les incapacités et le désavantage social}

Les travaux de P. Wood (1989) ont contribué à spécifier la distinction entre la maladie, la déficience qu'elle entraîne, les incapacités qui en résultent, et enfin le désavantage social qui en est la conséquence finale. Comme le souligne Denis Legay, un des intérêts de cette partition entre la maladie d'une part et les niveaux de son retentissement (avec un déficit de facultés élémentaires, des incapacités concernant des aptitudes plus générales, et enfin un désavantage social) d'autre part est de permettre de remarquer, dans le cas du handicap psychique, la possible réversibilité des rapports de dépendance entre ces niveaux de retentissement. Il s'agit en réalité d'enchaînements causaux entre la maladie et ses conséquences à différents niveaux, dont l'apparente réversibilité résulte en fait de leur circularité. Il est possible en effet, et peut-être même courant, que le désavantage social consécutif à la maladie (l'isolement social profond par exemple), aggrave en retour les incapacités (communicationnelles par exemple), qui retentissent sur les déficiences (des capacités discursives ou de l'organisation de la pensée) et finalement aggravent les troubles, et donc la maladie. L'intérêt d'admettre la possibilité d'une telle réversibilité des rapports de dépendance (ou circularité des liens causaux) entre la maladie et son retentissement fonctionnel et social apparaît dès lors clairement : en apportant une amélioration aux conséquences de la maladie, au désavantage social qu'elle induit, par exemple à l'isolement social, on peut en retour espérer un bénéfice sur certaines incapacités, et in fine sur la maladie elle-même. Cette perspective confère à la réhabilitation psychosociale un rôle accru. Elle ne se réduit pas à un rôle palliatif, visant à compenser telle ou telle incapacité et à réduire ainsi le désavantage social : ce faisant, elle permet d'obtenir en retour des effets bénéfiques sur certaines déficiences, et finalement sur la maladie elle-même.

\section{Le courant de la rébabilitation psychosociale et sa notion clé de rétablissement}

Le courant de la réhabilitation psychosociale, bien qu'il ait un caractère plus pratique que théorique, apparaît aujourd'hui comme l'expression dominante d'un abord (sinon d'un modèle) du handicap psychique affranchi du modèle médical. Il met l'accent sur les ressources de la personne et de son environnement pour dépasser les conséquences de la maladie, et en particulier sur le changement préalable de point de vue et de posture de l'individu pour mobiliser ces ressources. Pour cette raison, on peut argumenter qu'il 
s'apparente à un modèle existentiel du handicap et de son dépassement. Il importe en effet que ce changement de perspective soit adopté par l'usager lui-même pour être au principe du réenclenchement d'une dynamique, d'un changement de point de vue réouvrant la possibilité d'un projet de vie, et par là même la restauration de sens aux actes de la vie quotidienne et le recrutement de ressources pour surmonter certaines limitations.

Très schématiquement, la réhabilitation psychosociale est définie comme « un processus facilitant le rétablissement d'un individu à un niveau optimal de fonctionnement autonome dans la communauté.» (Cnaan et al., 1988). Elle met donc l'accent sur l'intégrité et sur les forces de l'individu plutôt que sur sa maladie. Le postulat sur lequel repose cette approche est que persiste chez tout individu, quel que soit le handicap, « une motivation à développer maîtrise et compétence dans des domaines de la vie qui vont lui permettre de se sentir indépendant et confiant en lui-même» (ibid). Le second postulat est que cette maîtrise et cette compétence peuvent être acquises ou augmentées par l'apprentissage.

Nous retiendrons des principes qui fondent la réhabilitation ceux qui concernent directement la réinsertion professionnelle :

- La priorité est donnée au développement et à l'optimisation des capacités de l'individu ( $1^{\text {er }}$ principe). Ce développement est compris comme un processus de croissance inhérent à la vie elle-même. Si le handicap psychique peut interférer avec ce processus, cela ne signifie pas qu'il l'abolit, comme on a tendance à le penser, en particulier les personnes handicapées elles-mêmes. C'est pour cette raison que ce développement des capacités doit être encouragé.

- L'apprentissage d'habiletés élémentaires pour agir dans un environnement social, vivre de façon indépendante, garder un emploi, etc., constitue donc un des moyens privilégiés de la réhabilitation ( $2^{e}$ principe $)$.

- L'autodétermination est conçue comme un droit des personnes à respecter, mais aussi comme une responsabilité à restituer à ces personnes et à promouvoir ( $3^{e}$ principe $)$.

La notion de handicap est traditionnellement comprise comme le fait de déficits et d'incapacités, qui entraînent une restriction des possibilités d'action et de vie sociale, ainsi que des formes de dépendance qui accroissent le sentiment de perte d'un contrôle sur sa propre vie. Pour rompre avec cette spirale de la dépendance et de la dépossession de soi, le principe d'autodétermination apparaît comme une exigence qu'il faut à la fois respecter et promouvoir chez des personnes à qui ce pouvoir a été longtemps dénié.

- Il est notable que parmi les principes de la réhabilitation soit stipulée la valeur du travail comme vecteur d'intégration (principe 10: a workcentered process), mais aussi comme lieu de réalisation de soi. Il est donc admis que le travail (rémunéré, et autant que possible en milieu ordinaire) est un facteur de reconnaissance sociale, et à ce titre un facteur d'identité, elle-même condition d'une autonomie. 
- La réintégration sociale des personnes handicapées psychiques doit être réalisée, autant que possible, dans le milieu ordinaire, qu'il s'agisse de leurs activités professionnelles ou de loisirs. En effet, l'inconvénient de regrouper des personnes handicapées (dans des lieux adaptés de travail, de logement ou de loisirs) est que cela entretient une forme de discrimination, que ces personnes sont les premières à intérioriser. L'objectif est au contraire de les réintégrer dans la communauté, c'est-à-dire dans la vie ordinaire.

- L'exigence d'intervention précoce est justifiée par le processus d'aggravation rapide de la perte d'autonomie et de la désocialisation, lié aux durées prolongées de soins en institution, aux rechutes et aux ruptures itératives qu'elles entraînent avec les lieux d'insertion sociale (le travail, l'environnement relationnel).

- La spécificité du mode de soutien à la réhabilitation est soulignée, en stipulant l'importance d'un «engagement» de la part des intervenants, mais aussi une posture que nous qualifierons de «démédicalisée», qui n'est plus celle d'un expert externe renforçant une asymétrie en termes d'autonomie. Dans cette ligne, le soutien des pairs est vivement encouragé.

Ces principes suffisent à indiquer la priorité accordée à une série de facteurs et dimensions : les capacités de l'individu qu'il s'agit d'optimiser, l'apprentissage des habiletés élémentaires, l'autodétermination.

La spécificité de cette approche peut être appréhendée à travers une de ses notions clés, celle de rétablissement (recovery). Cette notion incarne également le renoncement au modèle médical, puisqu'elle vient justement prendre la place de la notion de guérison, comprise comme restitution ad integrum, retour à l'état antérieur à la maladie. En effet, viser le rétablissement suppose en quelque sorte d'accepter de renoncer à l'objectif de guérison, d'accepter les formes résiduelles de la maladie et les limitations qui en résultent : c'est justement en tenant compte de ces limitations que le sujet peut reconstruire un projet de vie réaliste, se fixer des objectifs attractifs, et ce faisant se «rétablir» dans une vie active, pourvoyeuse de satisfactions et socialement intégrée. Cette notion de rétablissement est aussi celle qui illustre le mieux l'exigence de prise en compte du point de vue du sujet, autrement dit d'un point de vue «en première personne», subjectif et existentiel, car elle repose précisément sur une transformation de ce «point de vue», comme l'exprime, à partir de sa propre expérience, Patricia Deegan (1988) : "Being in recovery means that I know that I have certain limitations and things I can't do. But rather than letting these limitations be an occasion for despair and giving up, I have learned that in knowing what I can't do, I also open upon the possibilities of all the things I can do."

Dans les nombreuses publications sur l'expérience du rétablissement, on retrouve un certain nombre de constantes, bien synthétisées par Hélène Provencher (2002) :

- Le rétablissement doit être compris prioritairement comme une expérience, comme une attitude face à l'existence. Il ne peut être défini normativement 
«de l'extérieur», mais doit toujours faire référence à l'expérience subjective de la personne.

- En tant qu'expérience singulière d'un sujet, le rétablissement s'opère également selon des formes singulières, selon des choix propres au sujet : «Les personnes utilisatrices ont souligné la subjectivité et la singularité de l'expérience du rétablissement. La personne en voie de rétablissement se transforme conformément à ses valeurs et selon son propre rythme. Des changements se manifestent entre autres par une re-découverte du soi et de ses nouvelles possibilités d'agir sur l'environnement, une responsabilité renouvelée face à la construction de projets de vie significatifs... » (ibid).

- Le processus de rétablissement s'apparente donc à une forme de réengagement dans un projet existentiel qui, en tant que projet, suppose la restauration du sentiment de pouvoir agir.

\section{La notion de résilience}

Mentionnons encore ici que la notion de résilience est un autre exemple d'attention portée aux ressources qui permettent à la personne de «dépasser» l'impact négatif et durable qu'aurait pu avoir sur elle le fait d'avoir présenté une pathologie mentale sévère et désocialisante. À nouveau, on s'écarte du modèle médical, dans la mesure où l'accent est mis non sur des «remèdes externes », mais justement sur les ressources propres de l'individu, sur sa dynamique interne de préservation et de développement de stratégies permettant une issue positive. L'enjeu, tant d'un point de vue pratique que théorique, est alors d'établir les facteurs de résilience, et notamment de repérer parmi ces facteurs ceux qu'il est possible de renforcer. Parmi ces facteurs de résilience, l'environnement humain de la personne (l'entourage familial, les pairs, les collègues de travail) joue un rôle important, et à ce titre doit être pris en compte et soutenu.

\section{- Remarques conclusives sur les dimensions déterminantes des possibilités de réinsertion professionnelle}

La principale thèse qui se dégage de ces études est donc qu'une évaluation de la dimension proprement psychopathologique ainsi qu'une évaluation des performances cognitives sont certes utiles mais très insuffisantes pour appréhender les principaux facteurs qui conditionnent le retour au travail. Nous avons mentionné qu'une série d'autres dimensions sont également à considérer, parmi lesquelles le rapport qu'entretient la personne avec le travail, mais aussi le «sentiment d'efficacité» pour surmonter les obstacles (Bandura, 1997), les capacités relationnelles et la compétence sociale. Mais le propre du courant de la réhabilitation psychosociale est d'insister sur les 
enjeux existentiels et subjectifs d'un retour au travail, que le sujet est seul à pouvoir spécifier.

En un sens, on peut s'étonner qu'il y ait lieu d'insister sur cette thèse qui, finalement, peut paraître de bon sens. Il demeure cependant un écart entre cette conclusion et les pratiques que nous connaissons d'évaluation et de soutien à la réinsertion professionnelle. Cet écart tient au fait que ces évaluations sont le plus souvent réalisées par des cliniciens, qui privilégient l'évaluation des dimensions dont ils sont les experts (la dimension psychopathologique), ou pour lesquelles ils disposent des instruments d'analyse appropriés (évaluation des performances cognitives). Ce biais d'expertise peut être en partie corrigé par des évaluations pluridisciplinaires, comprenant une évaluation «technique», en situation de travail, par un formateur par exemple. Il demeure que certaines dimensions restent sans doute insuffisamment prises en compte, par méconnaissance de leur importance ou par manque d'outils d'appréciation. L'évaluation du handicap et des possibilités de réinsertion est plus qu'une évaluation clinique et psychopathologique, elle requiert des compétences et une attention particulière à des dimensions évoquées ici mais qui restent à mieux faire connaître. 


\section{Bibliographie}

ADDINGTON J., ADDINGTON D., MATICKA-TYNDALE E. (1991), “Cognitive functioning and positive and negative symptoms in schizophrenia", Schizophrenia Research 5 (2), p. 123-134.

BANDURA A. (1997), Self-efficacy: The exercise of control, New York, NY, USA: W. H. Freeman \& Co, Publishers, Traduction française (2003), Autoefficacité. Le sentiment d'efficacité personnelle, Bruxelles, De Boeck.

BARREYRE J.-Y., MAKDESSI Y. (2007), «Handicap d'origine psychique et évaluation des situations », Recherche documentaire, Délégation ANCREAI Ile-de-France - CEDIAS.

BARREYRE J.-Y., PEINTRE C. (2009), «Handicap d'origine psychique et évaluation des situations », Rapport volet II, Délégation ANCREAI Île-de-France - CEDIAS.

BEAULIEU A., MORIN P., PROVENCHER H., DORVIL H. (2002), «Le travail comme déterminant social de la santé pour les personnes utilisatrices de services en santé mentale», Santé mentale au Québec, Montréal, vol. XXVII, nº 1.

BELL M., KAPLAN E. A., BRYSON G. (1997), "Cognitive impairment and work behavior in schizophrenia", Schizophrenia Research, 24 (1-2), 219 p.

BRYSON G., BELL M., LYSAKER P., ZITO W. (1997), "The work behavior inventory: a scale for the assessment of work behavior for clients with severe mental illness", Schizophrenia Research, 24 (1-2), 220 p.

BURNS T et al. (2007), "The effectiveness of supported employment for people with severe mental illness: a randomised controlled trial”, Lancet, 370, p. 1146-1152.

CASTRA D. (2003), L'insertion professionnelle des publics précaires, Paris, Presses universitaires de France.

CHARBONNEAU C. (2002), «Développer l'intégration au travail en santé mentale, une longue marche à travers des obstacles sociopolitiques », Santé mentale au Québec, 27, 1, p. $158-176$.

CLOT Y. (1999), La fonction psychologique du travail, Paris, Presses universitaires de France.

CNAAN R. A., BLANKERTZ L. E., MESSINGER K., GARDNER J. R. (1988), "Psychosocial rehabilitation: Toward a definition", Psychosocial Rehabilitation Journal, 11 (4), p. 61-77.

CORBIÈRE M., BORDELEAU M., PROVOST G., MERCIER C. (2002), «Obstacles à l'insertion socioprofessionnelle de personnes ayant des problèmes graves de santé mentale : données empiriques et repères théoriques », Santé mentale au Québec, 27, p. 194-217.

CORBIÈRE M., MERCIER C., LESAGE A.-D. (2004), "Perceptions of barriers to employment, coping efficacy, and career search efficacy in people with mental health problems", Journal of Career Assessment, 12, p. 460-78.

DEEGAN P. E. (1988), "Recovery : the lived experience of rehabilitation", Psychosocial Rehabilitation Journal, 11, p. 11-19.

DEJOURS C. (2005), «Centralité du travail et santé mentale», Pratiques en santé mentale, 1, p. 22-28. 
EVANS J. D., BOND G. R., MEYER P. S., HEA WON KIM., LYSAKER P. H., GIBSON P. J., TUNIS S. (2004), "Cognitive and clinical predictors of success in vocational rehabilitation in schizophrenia", Schizophrenia Research,70, Issue 2, p. 331-342.

FOUGEYROLLAS P. (2002), «L'évolution conceptuelle internationale dans le champ du handicap : Enjeux sociopolitiques et contributions québécoises», Pistes, Vol. 4, № 2.

GREEN M. F. (1996), «What are the functional consequences of neurocognitive deficits in schizophrenia?», Am. J. Psychiatry, 153, p. 321-330.

HEATON R. K., GLADSJO J. A., PALMER B. W., KUCK J., MARCOTTE T. D., JESTE D. V. (2001), "Stability and course of neuropsychological deficits in schizophrenia", Arch. Gen. Psychiatry, 58, p. 24-32.

HOFFMANN H., KUPPER Z. (1997), "Relationships between social competence, psychopathology and work performance and their predictive value for vocational rehabilitation of schizophrenic outpatients", Schizophrenia Research, 23, p. 69-79.

LAUZON G., CHARBONNEAU C. (2001), «Favoriser l'intégration au travail : l'urgence d'agir», Association québécoise pour la réadaptation psychosociale, Québec.

LAUZON G., LECOMTE Y. (2002), «Rétablissement et travail», Santé mentale au Québec, vol. XXVII, n 1 , Montréal.

LECOMTE T., LECLERC C. (2004), Manuel de réadaptation psychiatrique, Québec, Presses de l'université du Québec.

LEGAY D. (2001), «Les pratiques françaises de réadaptation sociale», Information psychiatrique, 77 , p. 259-263.

LHUILIER D. (2006), Cliniques du travail, Erès.

LIBERMAN R. P., KOPELOWICZ A. (2005), "Recovery from Schizophrenia: a concept in search of research", Psychiatric Services, 56, 6, p. 735-742.

LYSAKER P., BELL M. (1995), "Work rehabilitation and improvements in insight in schizophrenia”, J Nerv Ment Dis, 183, p. 103-106.

MCGURK S. R., MELTZER H. Y. (2000), "The role of cognition in vocational functioning in schizophrenia", Schizophrenia Research, 45, p. 175-184.

MCGURK S. R., MUESER K. T. (2004), "Cognitive functioning, symptoms, and work in supported employment: a review and heuristic model", Schizophrenia Research, 70, p. 147-73.

PACHOUD B., NASCIMENTO STIEFFATRE M. (2005), «Psychopathologie et réinsertion professionnelle. Une clinique pluridimensionnelle requérant des ressources théoriques pluridisciplinaires », Clinique méditerranéenne, 72, p. 147-171.

PACHOUD B., LEPLEGE A., PLAGNOL A. (2008), Handicap psychique et réinsertion professionnelle, Rapport final du séminaire interdisciplinaire, Mire-DRESS.

PROVENCHER H.-L. (2002), «L'expérience du rétablissement : Perspectives théoriques », Santé mentale au Québec, XXVII (1), p. 35-64.

STIP E., CARON J., RENAUD S., PAMPOULOVA T., LECOMTE Y. (2003),

"Exploring cognitive complaints in schizophrenia: the subjective scale to investigate cognition in schizophrenia", Compr Psychiatry, 44, p. 331-40.

TORRENTE J. (2004), Le psychiatre et le travailleur. Cheminement de la psychopathologie du travail d'hier à demain, Paris, Doin.

VIDON G. (ed.) (1995), La réhabilitation psychosociale en psychiatrie, Paris, Éditions Frison-Roche. 
VIDAL-NAQUET P. A., MOLHO N., EYRAUD B. (2007), L'insertion professionnelle des personnes souffrant de troubles psychiques, Document du réseau Galaxie. En ligne : http://www.reseau-galaxie.fr/upload/modulepublication/cerpe_galaxie_fse.pdf

WILDER-WILLIS K. E., SHEAR P. K., STEFFEN J. J., BORKIN J. (2002), “The relationship between cognitive dysfunction and coping abilities in schizophrenia", Schizophrenia Research, 55 (3), p. 259-267.

WOOD P. H. N. (1989), "Measuring the Consequences of Illness", World Health Statistics Quaterly, 42, p. 115-121.

WORLD HEALTH ORGANIZATION (2000), Mental health and work: impact, issues and good practices, Geneva $(\mathrm{CH}), \mathrm{WHO}$.

ZAKZANIS K. K., HEINRICHS W. (1999), "Schizophrenia and the frontal brain: a quantitative review”, J. Int. Neuropsychol. Soc., 5, p. 556-566. 